\title{
Simulation of surface reactions
}

\author{
Michael Frenklach \\ Department of Mechanical Engineering, University of California at Berkeley, Berkeley, \\ CA 94720-1740, USA
}

\begin{abstract}
Advances in high-temperature material synthesis and processing necessitate better understanding of underlying surface processes. Both physical and chemical transformations are of concern, those underlying surface reconstructon, film growth, and material etching. While classical thermodynamic description may suffice for some aspects, dynamic evolution and coupling to reactant flow is of growing practical importance. The scientific challenge is to explain macroscopic phenomena in terms of atomistic processes. This manuscript reviews a theoretical approach for going from an atomistic level to mesoscale description to macroscale phenomena. The methods includes quantum-mechanical calculations of surface models, time-dependent Monte Carlo simulations using reaction probabilities derived from the quantum-mechanical calculations, and kinetic modeling parameterized to the Monte Carlo results. The examples are drawn from the fields of silicon, diamond, and carbon materials, stressing the methodology and emphasizing general features revealed by recent numerical simulations.
\end{abstract}

\section{INTRODUCTION}

Advances in high-temperature material synthesis and processing necessitate better understanding of underlying surface processes. Surface smoothness and texture of deposited films, deposition rate and efficiency, material etching, and formation of nanostructures are just a few familiar examples where knowledge of surface processes is required. While both physical and chemical transformations are of concern, the present discussion is focused on ability to understand and model chemical reactions, i.e., surface transformations that involve making and breaking of molecular bonds.

Chemical reactions occurring on a solid surface can be classified as follows: (a) chemisorption-adsorption of a gaseous precursor with the formation of a chemical bond; (b) desorption-the reverse of adsorption; (c) lattice building -incorporation of an adsorbate into the lattice of the solid; and (d) surface migrationtime-coordinated making and breaking of chemical bonds with the net effect equivalent of surface diffusion. For systems such as pure metals, where the exposed surface and the gas-phase growth species are all comprised of metal atoms, the chemical reactions are relatively simple and the behavior can be understood in terms of coordination number $(1,2)$. Material systems such as chemical vapor deposition (CVD) of silicon films are likely to fall into this category as well, because silicon surface looses its hydrogen atoms upon heating to CVD temperatures and a variety of Si-containing gas-phase species can grow the film upon collision with the surface (3).

On the other hand, chemical vapor deposition of diamond is complicated by the fact that diamond surface is covered with hydrogen atoms even at CVD temperatures, and therefore incorporation of carbon atoms from gaseous precursors into the diamond lattice requires a series of rather nontrivial chemical steps. In this particular case, the nature and rates of chemical reactions determine not just the rate or texture of film deposition, but whether the deposited material will be diamond. Materials like silicon carbide and gallium nitride, whose surface chemistry is still essentially unknown, will probably fall into the same category. 
The objective of the present discussion is to review theoretical approaches to study surface reactions, focusing on going from an atomistic level to mesoscale description to macroscale phenomena. The suggested methods include quantum-mechanical calculations of surface models, time-dependent Monte Carlo simulations using reaction probabilities derived from the quantum-mechanical calculations, and kinetic modeling parameterized to the Monte Carlo results. The examples are drawn from the fields of silicon, diamond, and carbon materials, stressing the methodology and emphasizing general features revealed in recent numerical simulations.

\section{THERMODYNAMIC DESCRIPTION}

With detailed information on surface reactions being often very scarce, some resort to thermodynamic description of surface-gas interfaces. Being applicable perhaps for simple, atomic metal systems, such approach is non valid on a global scale for systems like diamond CVD where neither equilibrium between the depositing solid and gas-phase species nor that among gas-phase species themselves is maintained (4). However, even though the entire system is not in equilibrium, some specific processes or individual reactions may be in a state of partial equilibrium, which is characterized by the forward and reverse fluxes balancing each other. An example of this is surface migration on diamond (100) surfaces, where transformations between surface radicals occur orders of magnitude faster than the rate-limiting attack by gaseous hydrogen atoms of the surface reactive sites (5). A consequence of such a situation is that a constrained equilibrium approach may provide some useful overall correlations. In this approach, the equilibrium between a selected group of species or phases is solved while keeping another group of species at prescribed levels. For example, in the case of diamond CVD, attempts have been made to express the process as equilibrium between diamond $s p^{3}$ and graphitic $s p^{2}$ phases constrained to the concentration of atomic hydrogen (6). Such modeling, however, relies on prior knowledge, the rate-limiting role of hydrogen atoms in diamond CVD, which can be revealed only in detailed kinetic simulations (7).

\section{GLOBAL KINETIC MODELING}

The dynamics of chemical transformations can be captured by reducing the entire process to a single or a small number of overall reaction steps (8), such as that used sometimes in modeling of silicon CVD,

$$
\mathrm{SiH}_{4} \rightarrow \mathrm{Si}_{\text {solid }}+2 \mathrm{H}_{2}
$$

These global reaction steps represent conservation of mass, with the reaction rate constants fitted to experiment or to detailed kinetic models. With proper parametrization of the rate constants, such models can be useful for coupling with fluid-dynamic codes to simulate a CVD process. It is usually difficult, however, to obtain high-quality fits over large ranges of experimental conditions.

\section{DETAILED KINETIC MODELING}

A more complete description of reaction kinetics is provided by the approach referred to as detailed kinetic modeling (DKM). In this method, the formation and consumption of each reacting species is described by a differential equation composed of the rates of the corresponding elementary chemical reactions, and the entire set of these equations is then integrated, usually numerically. A set of elementary reactions accounting for species transformations comprise what is called a reaction mechanism. The mechanism is developed (or postulated) based on the information available. Detailed kinetic modeling is a well-developed method for gas-phase systems and has found extensive use in the fields of combustion and atmospheric chemistry; it is now being applied for description of the gas phase in chemical vapor deposition.

The DKM approach has also been applied to surface reaction systems (7,9-13). There are two problems associated with this approach: the lack of knowledge of elementary reactions occurring on surfaces, and inability to account for steric factors. The former presents a scientific challenge while the latter indicates the principal limitation of the DKM method to model surface reactions. 
Various experimental and theoretical methods have been applied to study surface reactions. However, most current experimental techniques lack spatial resolution required to identify individual atomic-scale events and the most accurate theoretical (quantum ab initio) methods are computationally formidable for modeling large (more than about 10 heavy atoms) atomic systems required to represent the surface. In the area of diamond CVD it was suggested (14-16), as a first approximation, to think of surface reactions as those of large alkane molecules. This postulate of chemical similarity between analogous surface and gasphase reactions was founded on the premise that the chemical reactivity of solid carbonaceous materials is localized at the carbon sites in a manner similar to that of the corresponding gaseous species. This approach led to correct prediction of diamond film growth rates in numerous deposition reactors (13) and found support in experimental $(17)$ and theoretical $(18,19)$ results obtained for some elementary reactions. However, as mentioned above, this type of modeling does not have a provision to consider steric factors associated with surface kinetics, like the specific character of neighboring sites and surface diffusion. For instance, the outcome of a reaction event occurring at a given surface site often depends not only on the chemical nature of this site but also on its location (e.g., in-plane versus edge) and the number and nature of its neighboring sites. One way of addressing this problem is to specify all possible species, each representing, in addition to the chemical nature, a different steric situation. However, this would lead to an excessive number of surface species to consider and, more importantly, make it unclear how to separate a given surface into individual species and to account properly for the evolution of their surface number densities. Thus, in summary, while DKM models can be used for description of localized surface reactions, they cannot, in principle, describe the formation of a film or assess development of surface morphology.

\section{KINETIC MONTE CARLO SIMULATIONS}

The limitation of detailed kinetic modeling to account for steric factors can be resolved by the method known as kinetic Monte Carlo (KMC), also referred to as time-dependent or dynamic Monte Carlo. In this approach, the process is modeled as a Markovian sequence of reaction events, which include both bimolecular gas-surface collisions and unimolecular transformations of surface species. Each type of possible reactions is associated with a reaction probability and their complete set determines the time sequence of reaction events.

Several approaches to numerical implementation of KMC simulations have been proposed (see $(20,21)$ for references). Among them, the one of Gillespie (22) is physically rigorous, chemically consistent, and easily adaptable to surface kinetics. To illustrate this approach, let us consider a surface containing $s$ reactive sites, each site may undergo $N_{i}$ reactions $(i=1,2, \ldots, s)$, and for each reaction the per-site rate is $k_{j}$ $\left(j=1,2, \ldots, N_{i}\right)$. The per-site rate is specified by the first-order rate constant for an unimolecular surface reaction and by the product of the second-order rate constant (or, equivalently, reaction probability times collision frequency) and the concentration of the colliding partner for a bimolecular gas-surface reaction.

Given a particular state of surface at an instant of time $t_{n}$, we first calculate the time interval of the next reaction event to take place at each surface site

$$
\Delta t_{i}=\frac{1}{\sum_{j=1}^{N_{i}} k_{j}} \ln r
$$

where $r$ is the random number distributed uniformly from 0 to 1 and the summation of $k_{j}$ represents the total reaction rate at site $i$. The site corresponding to the smallest $\Delta t_{i}$ is the one at which reaction takes place next at time

$$
t_{n+1}=t_{n}+\min \left\{\Delta t_{1}, \Delta t_{2}, \ldots, \Delta t_{s}\right\}
$$

and the specific reaction event that takes place at this site is determined by a random selection among probabilities of individual reactions at this site, determined as 


$$
p_{j}=\frac{k_{j}}{\sum_{j=1}^{N_{i}} k_{j}} ; j=1,2, \ldots, N_{i}
$$

Kinetic Monte Carlo methods, in one form or another, have been widely used for studying surface phenomena. One large class of problems addressed is the time development of the surface interfaces (23). These studies largely focus on scaling properties of interface roughening and typically employ so-called "toy models", with generic overall rate parameters (24) applicable to metals (25). A similar approach has also been applied to adsorption-desorption phenomena, those describing activation of surface (26), catalysis (27), and interpretation of temperature-programmed desorption experiments (28).

Recently, KMC methods begun to be employed to study detailed mechanisms of surface reaction systems such as diamond film growth $(20,29)$. The difficulty here rests in the vastly differing time scales that need to be considered, and this slows down the computations to the extent that such simulations may become impractical. This situation typically arises because many steps of the assumed reaction mechanism proceed through formation of relatively unstable, short-lived intermediates whose decomposition and transformation rates are orders of magnitude faster than the gas-surface collision rates. One common solution is to remove the small reaction time scales by assuming the short-lived intermediates to be in the steady state. The overall rate expressions resulting from this assumption are then used as $k_{j}$ in the calculations of reaction probabilities ( 30$)$, as described above.

\section{MOLECULAR DYNAMICS SIMULATIONS}

Both $\mathrm{KMC}$ and DKM methods rely on the prior knowledge of reaction mechanisms and associated rate parameters. Development of detailed reaction mechanisms is in itself a challenging task, in which theoretical approaches will probably play a critical role. One of such methods, which is becoming increasingly popular and beginning to provide useful information for gas-surface reactive collisions and surface transformations, is molecular dynamics (MD) simulation. In this approach (31), trajectories of atoms representing the surface and gaseous colliders are simulated by numerically integrating Newton's equations of motion. The initial conditions of both surface and incident molecules are readily specified, and the results of computer simulations provide a microscopic view of the collision leading to adsorption and surface-transformation dynamics.

Methods of molecular dynamics are being continuously developed. The principal difference between various methods lies in the way interatomic forces are calculated. The two main approaches are based on empirically-parametrized potentials and on quantum-mechanical ones.

Empirical potentials (EP) are developed by first choosing an appropriate analytic functional form containing adjustable parameters. Most often the potential energy is approximated as the sum of twobody interactions between atoms (32), although three-body terms are frequently included to account for directional bonding. The parameters are then optimized, fitting the potential to experimentally determined structures and energetics, equilibrium properties, or to the results of quantum mechanical calculations on model clusters. Dynamic simulations utilizing empirical potentials are relatively inexpensive in terms of computation time, making possible trajectory calculations for thousands of atoms up to the nanosecond time scale. Many EP potentials have been developed for MD simulations of solids, and a wide range of experimental processes and properties of the condensed phases have been investigated using these potentials.

There are problems, however, inherent in the application of empirical potentials to the study of chemical reactions. For one, the task of parameterization becomes intractable as the number of elements included in the system increases. While the development of two- and three-body potentials for surface reactions within two-element systems appears to be feasible, a three element system, as with silicon-carbonhydrogen, would theoretically require fitting all parameters included in 6 two-body terms and 18 threebody terms. 
A more fundamental problem arises when trying to develop a potential to describe atomic geometries with differing electronic structure. Because empirical potentials are represented as interactions between atoms, electronic structure is implicitly incorporated into the parameters and reflects that of the structure or compound to which the potential has been fit. For example, the difficulty encountered in developing a single potential energy function to universally describe the properties of crystalline silicon and silicon microclusters has been demonstrated and discussed (33). In the case of chemical reactions, the processes of bond formation and bond breaking traverse regions of the potential energy surface which cannot be adequately fit unless directly parameterized at the expense of equilibrium structures. For instance, molecular dynamics simulations using empirical potentials to study the etching of silicon surfaces by fluorine have been recently criticized (34) because the electronic structure for $\mathrm{Si}-\mathrm{F}$ bond formation was not physically realistic, leading to predicted etching mechanisms inconsistent with experiment.

Thus, while offering computational speed and thus being suitable for building up the reaction statistics for well understood systems, the use of empirical potential limits the applicability of molecular dynamics in exploratory investigations where one is interested in testing unknown reactions, surface rearrangements, and transition states. This is especially so when the chemical reaction may involve recrossing of potential energy surfaces or when dealing with a multi-element systems, for which the development of an empirical fit may become in itself overwhelming.

An ideal approach in such situations would be to calculate the forces from an ab initio quantum-mechanical potential, which utilizes the exact electronic Hamiltonian that contains a description of the potential energy due to the interaction of electrons with nuclei and other electrons and the kinetic energy of the electrons. Unfortunately, such calculations usually require excessively large amounts of computational effort and, at present, one cannot obtain a high-level ab initio description of atomic systems sufficiently large to represent surfaces. Attempts of using ab initio potentials in molecular-dynamics calculations have been limited to small molecular systems (35), tight-binding minimal basis sets (36), or the combination of pseudopotentials and plane wave bases [the method referred to as Car-Parrinello (37)] describing only the ground state of the potential energy surface.

Quantum semiempirical (QS) potentials offer a compromise between high-level quantum description and computational speed. Over the past thirty years or so, QS techniques have been developed as computationally more efficient alternatives to ab initio methods (38). Remaining within the basic quantum mechanical framework, QS methods make extensive use of approximation and experimental parameterization in determining electronic interactions, that emphasizes the chemical nature of the interaction, i.e., localized bond formation and bond breaking. While the accuracy of ab initio calculations is controlled by the choice of basis set used to represent electronic wave functions and the level of electron correlation, the accuracy of semiempirical methods relies on the validity of approximation and deftness of parameterization.

QS calculations can be several orders of magnitude faster than ab initio techniques, yet they do not approach the speed provided by empirical potentials. Several groups have recognized the advantages of combining the more complete chemical description of semiempirical quantum-chemical potentials and the efficiency of empirical potentials for dynamic simulation of enzymes (39), reactions in solution (40), and surface reactions (41). In the latter approach, a portion of the surface interacting with an incident molecule or representing a surface transformation is described by a QS potential and is embedded in an environment of empirically described atoms allowing transfer of energy to and from the reaction site in a physically meaningful manner. Simulating the chemical reaction on a quantum-chemical level, this combined-potential format has the advantage of making model surfaces large enough to allow more complete reproduction of vibrational density of states, more authentic dissipation of the energy released upon bond formation at the surface, and most importantly to mechanism identification, to reproduce more realistically the energetic and steric constrains of the surface (as opposed to a free atomic cluster).

Figure 1 depicts en example of such a combined surface model used in MD simulations of the reaction between an acetylene molecule and a $\mathrm{Si}(100)-(2 \times 1)$ surface $(41)$. The $\mathrm{Si}(100)$ surface was modeled by 360 silicon atoms placed in the diamond structure to form 10 layers with 36 atoms each. Periodic boundary conditions were implemented by the minimum image convention in two dimensions across planes normal 
to the $\langle 110\rangle$ and $\langle 1 \overline{1} 0\rangle$ directions. Gray shaded atoms shown in the top four layers of Fig. 1 represent a $\mathrm{SQ}$ region surrounded by EP atoms, shown white. Cross-hatched atoms, in layers 7-9 below the surface, were also treated empirically and served as a heat bath to control simulation temperature. The bottom layer atoms, shown black in Fig. 1, were held at fixed bulk positions throughout the MD simulations.

Subroutines from the MOPAC quantum chemical program package (42) were incorporated into a molecular dynamics program to calculate the potential energy and interatomic forces in the SQ region including the gas-surface interaction. The MOPAC package gives access to several semiempirical Hamiltonian which are currently parameterized for a large number of first and second row elements, and provides information on electronic structure, allowing changes in bond order, charge population, and spin density on atomic orbitals to be followed throughout the course of a simulation.

At each dynamics time step, the atoms making up the SQ region were treated as a static molecular cluster. The lowest electronic energy state of this instantaneous nuclear configuration was determined by the unrestricted Hartree-Fock self-consistent field method. The AM1 parameterization (43) using Slater-type orbitals and minimal basis sets was employed. The potential energy governing the motion of these atoms thus included contributions from electron-electron, electron-nuclear, and nuclear-nuclear interactions and the electron kinetic energy.

Figures 2 and 3 demonstrate results that one obtains with this type of calculations. Figure 2 depicts formation and breaking of chemical bonds between $\mathrm{C}$ and $\mathrm{Si}$ atoms during an acetylene molecule attack of a silicon surface, while Fig. 3 shows a mobile precursor state in another MD simulation. Similar calculations for diamond surfaces have identified new classes of surface reactions (5). Figure 4, taken from one of the latest studies (44), demonstrates that the combined-force MD technique is capable of predicting vibrational spectra comparable to experiment.

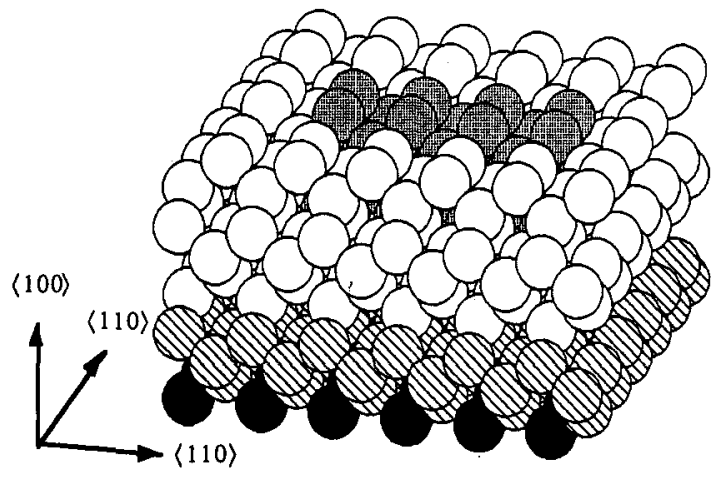

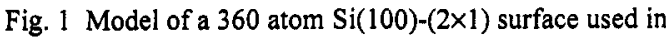
combined-force MD simulations (41). Shaded spheres represent quantum mechanically described atoms, white spheres, empirical atoms, cross hatched spheres, empirical atoms subjected to temperature control as described in the text, and black spheres, empirical atoms held in fixed positions throughout the simulations.

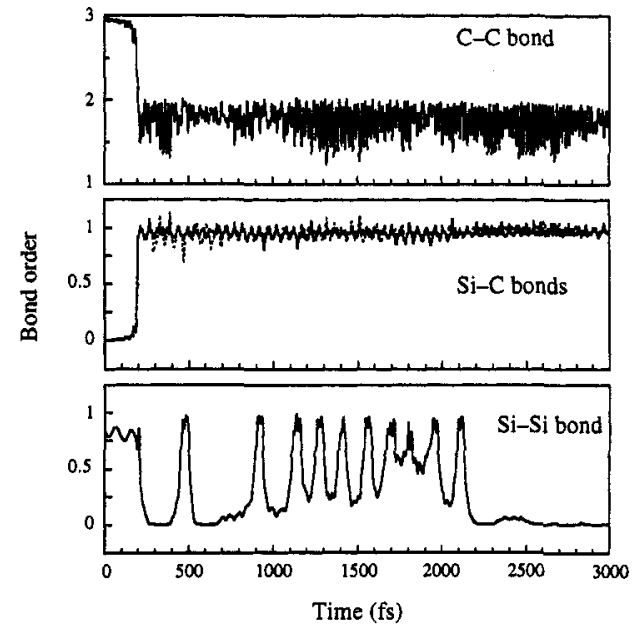

Fig. 2 Time evolution of bond orders obtained in a combined-force $M D$ simulation for a $\mathrm{C}_{2} \mathrm{H}_{2}$ molecule reacting with a $\mathrm{Si}(100)-(2 \times 1)$ surface dimer $(41)$. 


\section{SUMMARY: SUGGESTED APPROACH}

We thus see that each of the simulation methods has its strong and weak points. Thermodynamic description is the most straightforward but lacks dynamic information. Among dynamic modeling methods, global kinetic modeling is the simplest but mostly suitable for well established systems. Detailed kinetic modeling and kinetic Monte Carlo methods require prior knowledge of reaction mechanisms. And, finally, molecular dynamics simulations, even with simplest potentials, are computationally intensive and at present can only be used for reaction times up to nanoseconds, whereas many surface reactions of interest occur on a time scale of micro- and milliseconds.

It does not mean, however, that one cannot approach modeling of surface reactions on rigorous scientific grounds; it just means that one has to use a combination of modeling methods to accomplish this. Considering the advantages of different methods, the current computational capability, and the present scientific challenge of explaining macroscopic phenomena in terms of atomistic processes, the following approach may be suggested for going from an atomistic level to mesoscale description to macroscale phenomena: use of quantum-mechanical calculations of surface models, static or dynamic, to obtain energetic and structural information for different reaction possibilities; transition-state-theory (TST) evaluation of reaction rates based on the quantum-mechanical information; kinetic Monte Carlo simulations using reaction probabilities derived from the quantum-mechanical and TST calculations; and detailed kinetic modeling parameterized to the Monte Carlo results.

\section{ACKNOWLEDGMENTS}

The ideas presented in this manuscript were developed while working over the years on diamond CVD projects supported by the Innovative Science and Technology Program of the Ballistic Missile Defense Organization via the U.S. Office of Naval Research, Contract No. N00014-92-J-1420, and the National Science Foundation, Grant No. CTS 9404499.

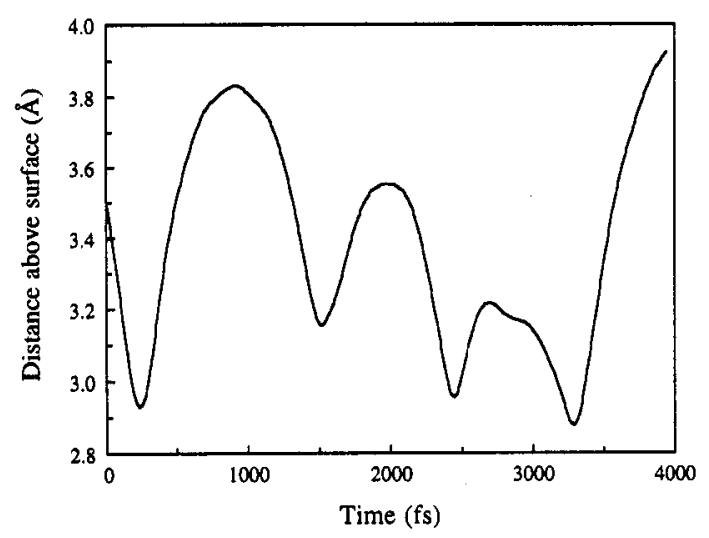

Fig. 3 Bouncing motion of a $\mathrm{C}_{2} \mathrm{H}_{2}$ molecule trapped in a physisorbed state above a $\mathrm{Si}(100)-(2 \times 1)$ surface $(41)$. The distance above the surface is defined as the difference between the $\mathrm{C}_{2} \mathrm{H}_{2}$ center of mass and the plane of the surface.

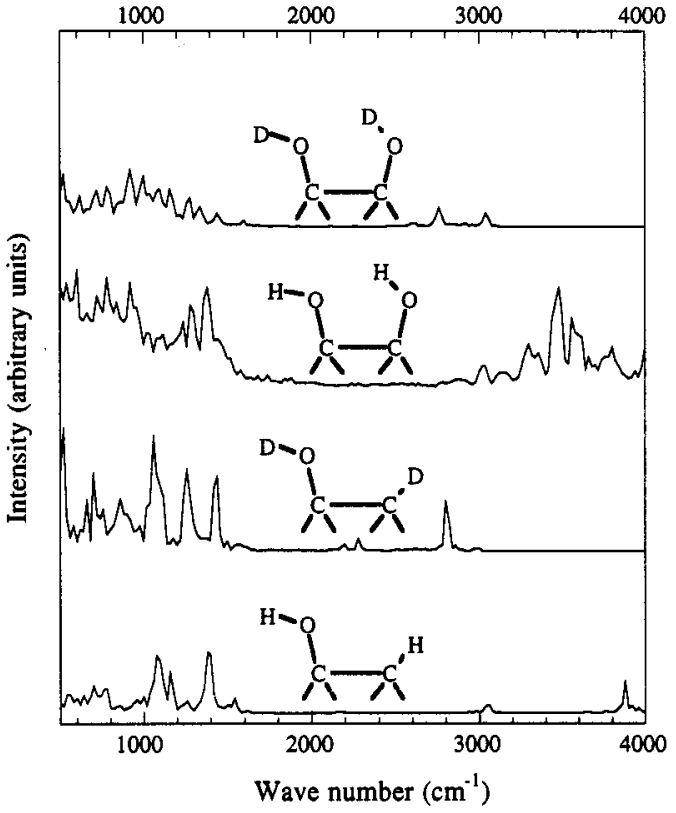

Fig. 4 Vibrational spectra of a diamond (100)-(2×1) surface saturated with hydroxyl groups computed in a combined-force isothermal MD simulation of $1 \mathrm{ps}$ duration at $300 \mathrm{~K}(44)$. 


\section{REFERENCES}

1. M. Boudart and G. Djéga-Mariadassou. Kinetics of Heterogeneous Catalytic Reactions, Princeton University, Princeton, NJ (1984).

2. S. R. Morrison. The Chemical Physics of Surfaces, Plenum, New York (1990).

3. J. M. Jasinski, B. S. Meyerson and B. A. Scott. Annu. Rev. Phys. Chem. 38, 109 (1987).

4. K. E. Spear and M. Frenklach. In Synthetic Diamond: Emerging CVD Science and Technology (K. E. Spear and J. P. Dismukes, eds.), p. 243, Wiley, New York (1994).

5. M. Frenklach and S. Skokov. J. Phys. Chem. 101, 3025 (1997).

6. W. A. Yarbrough. J. Electrochem. Mater. 20, 133 (1991).

7. M. Frenklach and H. Wang. Phys. Rev. B 43, 1520 (1991).

8. M. Frenklach. In Numerical Approaches to Combustion Modeling (E. S. Oran and J. P. Boris, eds.), p. 129, American Institute of Astronautics and Aeronautics, Washington, D.C. (1991).

9. K. F. Jensen, D. I. Fotiadis and T. J. Mountziaris. J. Crystal Growth 107, 1 (1991).

10. Y.-F. Wang and R. Pollard. J. Electrochem. Soc. 142, 1712 (1995).

11. S. J. Harris and D. G. Goodwin. J. Phys. Chem. 97, 23 (1993).

12. M. E. Coltrin and D. S. Dandy. J. Appl. Phys. 74, 5803 (1993).

13. B. W. Yu and S. L. Girshick. J. Appl. Phys. 75, 3914 (1994).

14. M. Frenklach and K. E. Spear. J. Mater. Res. 3, 133 (1988).

15. M. Frenklach. J. Appl. Phys. 65, 5142 (1989).

16. M. Frenklach. In Carbon in the Galaxy: Studies From Earth and Space (J. C. Tarter, S. Chang and D. J. DeFrees, eds.), p. 259, NASA Conference Publication 3061 (1990).

17. L. N. Krasnoperov, I. J. Kalinovski, H. N. Chu and D. Gutman. J. Phys. Chem. 97, 11787 (1993).

18. P. de Santa Claire, P. Barbarat and W. L. Hase. J. Chem. Phys. 101, 2476 (1994).

19. P. de Santa Claire and W. L. Hase. (1997, to be published.).

20. M. Frenklach. J. Chem. Phys. 97, 5794 (1992).

21. E. J. Dawnkaski, D. Srivastava and B. J. Garrison. J. Chem. Phys. 102, 9401 (1995).

22. D. T. Gillespie. J. Phys. Chem. 81, 2340 (1977).

23. A.-L. Barabási and H. E. Stanley. Fractal Concepts in Surface Growth, Cambridge University, New York (1995).

24. A. Zangwill. J. Crystal Growth 163, 8 (1996).

25. Z. Zhang and M. G. Lagally. Science 276, 377 (1997).

26. M. Frenklach. Phys. Rev. B 45, 9455 (1992).

27. H. C. Kang and W. H. Weinberg. Acc. Chem. Res. 25, 253 (1992).

28. A. P. J. Jansen. Comput. Phys. Commun. 86, 1 (1995).

29. E. J. Dawnkaski, D. Srivastava and B. J. Garrison. J. Chem. Phys. 104, 5997 (1996).

30. M. Frenklach. In Twenty-Sixth Symposium (International) on Combustioneds.), p. 2285, The Combustion Institute, Pittsburgh, PA (1996).

31. J. M. Haile. Molecular Dynamics Simulation, Wiley, New York (1992).

32. D. W. Brenner and B. J. Garrison. Adv. Chem. Phys. 76, 281 (1989).

33. H. Balamane, T. Halicioglu and W. A. Tiller. Phys. Rev. B. 46, 2250 (1992).

34. P. C. Weakliem, C. J. Wu and E. A. Carter. Phys. Rev. Lett. 69, 200 (1992).

35. B. Hartke and E. A. Carter. Chem. Phys. Lett. 189, 358 (1992).

36. D. A. Drabold, R. Wang, S. Klemm, O. Sankley and J. Dow. Phys. Rev. B 43, 5132 (1991).

37. F. Buda, G. L. Chiarotti, R. Car and M. Parrinello. Phys. Rev. B 44, 5908 (1991).

38. J. J. P. Stewart. In Reviews in Computational Chemistry (K. B. Lipkowitz and D. B. Boyd, eds.), p. 45, VCH, New York (1990)

39. U. C. Singh and P. A. Kollman. J. Comput. Chem. 7, 718 (1986).

40. M. J. Field, P. A. Bash and M. Karplus. J. Comput. Chem. 11, 700 (1990).

41. C. S. Carmer, B. Weiner and M. Frenklach. J. Chem. Phys. 99, 1356 (1993).

42. J. J. P. Stewart. Quantum Chemistry Exchange Program No. 455, Indiana University, Bloomington, IN (1983).

43. M. J. S. Dewar, E. G. Zoebisch, F. Healy and J. J. P. Stewart. J. Am. Chem. Soc. 107, 3902 (1985).

44. S. Skokov, B. Weiner and M. Frenklach. Phys. Rev. B 55, 1895 (1997). 